Stephen S. L. Peppin

Robert W. Style 



\title{
The physics of frost heave and ice-lens growth
}

\author{
Stephen S. L. Peppin ${ }^{1}$
}

Robert W. Style ${ }^{2}$

\author{
April 16, 2012 \\ ${ }^{1}$ Oxford Centre for Collaborative Applied Mathematics, University of Oxford, \\ Mathematical Institute, 24-29 St. Giles', Oxford, OX1 3LB, UK \\ ${ }^{2}$ Department of Geology \& Geophysics, Yale University, New Haven, CT, 06520-8109, \\ USA
}

\begin{abstract}
The formation of rhythmic lenses of ice in freezing soils is an intriguing geophysical phenomenon that is not fully understood, despite much experimental and theoretical work over the past century. We review proposed mathematical models of ice lens growth and frost heave, from early capillary theories and models based on the concept of a frozen fringe, to more recent advances that have revitalised the capillary model. In addition we identify several key experimental and theoretical challenges that are still to be resolved.
\end{abstract}

\section{Introduction}

Frost heave refers to the upward displacement of the ground surface caused by the formation of ice lenses - discrete bands of ice that form in freezing soil. This phenomenon is partly responsible for beautiful surface patterns that appear in very cold, permafrost areas (figure 1). Similar patterned ground has been observed on ice-rich portions of Mars, and the observations have been used to draw interesting conclusions about previous climate conditions [1]. Frost heave also has many practical and industrial implications. Residents in cold countries are familiar with the annual appearance of frost-heave-induced road damage after winter cold spells, and the heaving forces are capable of damaging 


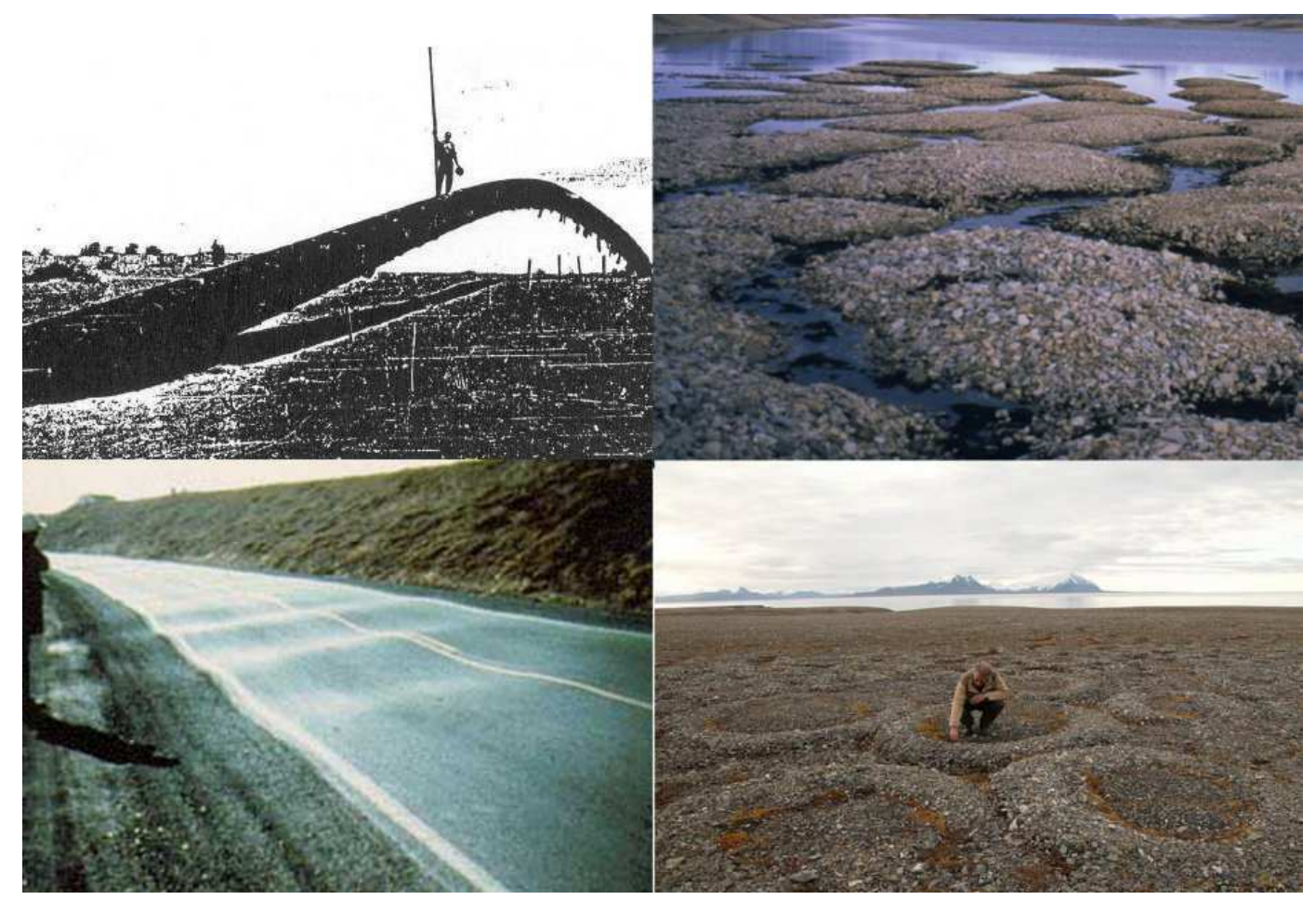

Figure 1: Phenomena caused by frost heave. Clockwise from top left: a pipeline heaved out of the ground [3], hummocks, stone circles and road buckling [2].

infrastructure such as pipelines, railways and buildings (figure 1). In the United States, over two billion dollars is spent annually repairing frost-heave damage to roads alone [2]. Thus worldwide, the cost is tremendous.

Despite the importance of frost heave, there are still many unanswered questions about the underlying mechanisms. Almost a century has passed since Stephen Taber demonstrated experimentally the basic features of frost heave $[4,5,6]$, and scientists are still actively working to understand his observations. Taber's key result was that frost heave is not, as commonly assumed, caused by the expansion of water upon freezing; he showed that a column of soil saturated with benzene (a liquid that contracts as it freezes) also experiences frost heave. Instead Taber demonstrated that frost heave is caused by the migration of water from lower, unfrozen regions of a soil column towards the freezing front. There, it deposits as bands of pure ice in the soil - ice lenses - which force the soil apart as they grow, heaving the surface upwards. This process can cause almost unlimited heave of the soil surface, provided there is a sufficient supply of water and slow enough freezing $[5,6,7]$.

Any quantitative theory of frost heave must explain two salient features of the phe- 
nomenon: the migration of water from lower, unfrozen regions of the soil to colder regions where it freezes as excess ice, and the tendency of the ice to force the soil apart and deposit as periodic ice lenses. In this work we review the various mathematical models that have been proposed since the 1930s. We classify the theories into two broad categories, capillary models and frozen-fringe models, choosing representative works for each, rather than attempting an exhaustive list of all published theories.

\section{Capillary theory}

\subsection{Suction of water towards the ice lens}

The first widely accepted explanation for frost heave was given by Taber [6] and later quantified by Beskow [7], Gold [8], Jackson et al. [9, 10] and Everett [11]. This theory relies on the Clapeyron equation describing thermodynamic equilibrium in a system at temperature $T$ and containing ice at pressure $P_{i}$ and water at $P_{w}[12,9,13]$ :

$$
P_{i}-P_{w}=\frac{\rho_{w} L_{f}}{T_{m}}\left(T_{m}-T\right) .
$$

Here $L_{f}$ is the latent heat of fusion at the bulk freezing temperature $T_{m}$ and atmospheric pressure $P_{a t m}$, and $\rho_{w}$ is the density of water. In fact this is not the complete version of the Clapeyron equation, as equation (1) neglects a term $\left(\rho_{w} / \rho_{i}-1\right)\left(P_{a t m}-P_{i}\right)$, where $\rho_{i}$ is the density of ice [11]. This term is typically small since $\rho_{w} \approx \rho_{i}$.

Figure 2(a) shows a simple system that illustrates the basic frost heave phenomenon [9]. There is a layer of ice above a water-saturated soil, which in turn sits on a reservoir containing water at pressure $P_{R}$. The whole system is isothermal with temperature $T<T_{m}$. The pore water and reservoir water remain unfrozen as the pores of the soil are sufficiently small that ice cannot invade; the soil acts like a semi-permeable membrane and stops the ice from entering the soil pores (Gibbs-Thompson effect, cf Section 2.2). This is illustrated schematically in figure 2(b) which shows a close-up of the ice-lens soil interface. Due to the overlying weight, the pressure of ice in the lens is $P_{o}$ (assumed isotropic [14]). Then equation (1) gives the water pressure necessary for equilibrium to be

$$
P_{c l}=P_{o}-\frac{\rho_{w} L_{f}}{T_{m}}\left(T_{m}-T\right),
$$

which we shall call the Clapeyron pressure. 


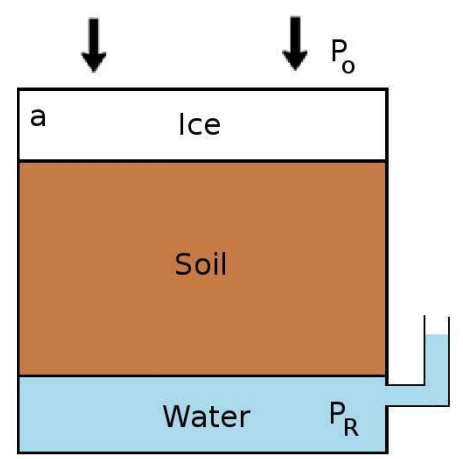

Isothermal system

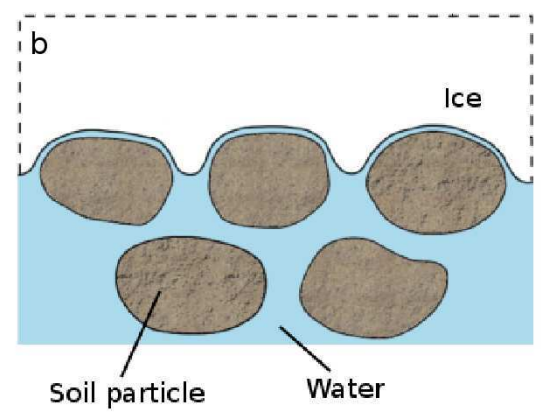

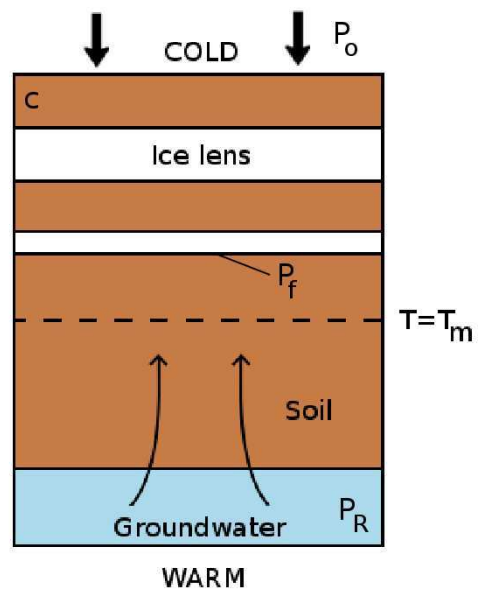

Figure 2: Schematic diagrams for the frost heave process. (a) A simple isothermal model of frost heave. (b) Microscopic view of the soil particles at the ice-soil interface. (c) A typical column of soil with multiple ice lenses. The soil is being frozen from the top down.

Suppose we initially fix the reservoir pressure $P_{R}=P_{c l}$ so the system is at equilibrium with no flow in the soil. If the temperature is reduced and $P_{o}$ held constant, then equation (2) shows that $P_{c l}$ decreases. If $P_{R}$ is held constant, there will be a pressure drop across the soil layer and water will flow from the reservoir toward the ice lens, where it will freeze onto the lens, causing it to grow. Experimental realizations of this system demonstrate that if the reservoir has a sufficient supply of water at $P_{R}$, the ice lens can grow indefinitely, heaving up the surface $[15,16,17]$. The experiments also confirm that the flow can be stopped by either reducing the reservoir pressure, or increasing the overburden pressure $P_{0}$, until the equilibrium condition $P_{R}=P_{c l}$ is satisfied $[15,16,17]$. This water flow, and the associated accumulation of extra ice at the freezing front represents the basic capillary theory explanation of frost heave.

The Clapeyron equation explains thermodynamically why lowering the temperature below freezing in figure 2(a) causes water to be sucked towards an ice front. In order for the ice lens to grow, however, it is necessary for water to attach to ice at the icelens/soil boundary. A conceptual difficulty arises when we consider the interface between the soil and the ice lens, as shown in figure 2(b). If the soil particles are frozen to the ice, then the only place that water can attach to ice is along the pore space between the soil particles, and this will not result in a thickening ice lens. A resolution to this problem was conjectured by Taber $[5,6]$. He suggested that microscopically-thin films of water exist between the ice and soil particle surfaces, and these allow water to flow around the 
soil particles and attach evenly to the growing ice lens. As later work has shown, these 'premelted' films do exist, and are caused by molecular interactions between soil particles and ice [18, 19].

\section{$2.2 \quad$ Ice entry and the maximum frost heave pressure}

A key assumption of the capillary theory is that ice does not immediately penetrate into the pores of the soil as the temperature drops below $T_{m}$. This is a result of the Young-Laplace equation for the pressure difference across a curved ice-water interface [20]

$$
P_{i}-P_{w}=\frac{2 \gamma_{i w}}{r},
$$

where $\gamma_{i w}$ is the ice-water surface energy and $r$ is the radius of ice (assumed to approximate a spherical cap) adjacent to a pore. This is shown schematically in figure 2(b). If $r$ is larger than the effective pore radius $r_{p}$, the ice cannot penetrate through the pore; ice can only invade once the pressure difference $\Delta P=P_{i}-P_{w}$ becomes sufficiently large that $r=r_{p}$. Thus ice invades the soil pores at the critical pressure difference

$$
\Delta P_{\max }=\frac{2 \gamma_{i w}}{r_{p}}
$$

The temperature $T_{p}$ at which ice invades the pores is found by combining (1) and (4) to obtain a form of the Gibbs-Thomson equation

$$
T_{p}=T_{m}\left(1-\frac{2 \gamma_{i w}}{\rho_{w} L_{f} r_{p}}\right) .
$$

In the capillary model frost heave stops once $T \leq T_{p}$, when ice is assumed to fill the soil pores, blocking them and preventing water being sucked up to the growing lens. This gives rise to a maximum frost-heave pressure, $P_{m}$, which is the largest ice pressure that can occur before pore entry. If we note that the liquid pressure in the water column will always be $\leq P_{R}$ during freezing, then we can obtain $P_{m}$ by combining equations (3) and (4) to give

$$
P_{m}=P_{R}+\frac{2 \gamma_{i w}}{r_{p}}
$$

The ideas expressed by equations (1)-(6) were tested extensively by early researchers and showed good quantitative agreement with experiments for monodisperse soils at temperatures close to $T_{m}[21,22,23,24,25,15,26,16,27]$. The simple isothermal capillary model can in principle be adapted to general soil-freezing situations such as that 
shown in figure 2(c). A drop in air temperature causes freezing of the soil and ice lens formation. At the leading edge of the warmest ice lens the pressure drops - as discussed above - and this causes water to be sucked up from warm $\left(T>T_{m}\right)$ groundwater to swell the lens, and heave the soil surface upwards. However, attempts to extend the capillary model to non-equilibrium situations led to some problematic shortcomings, as we discuss in the next section.

\subsection{Problems with the capillary theory}

Despite the ability of the capillary theory to explain the basic heaving phenomenon, significant deficiencies of the model became apparent in the 1960s and 1970s. Three major difficulties were identified by frost heave researchers.

\section{1) $P_{m}$ predictions deviated from experiments in polydisperse soils.}

Predictions of the maximum frost-heave pressure matched well with experiments on idealized soils composed of monodisperse particles $[22,23]$. However when soils containing a range of particle sizes were used, it was found that equation (6) did not agree with experimental results, as significantly larger heaving pressures were observed than were predicted $[28,29,30]$.

2) Break down of Clapeyron equation outside equilibrium. Capillary theory can in principle be used to predict the flow rate towards the lens as the soil is frozen: for a rigid soil with homogeneous pore size Darcy's law [31, 32] determines the flow rate towards the ice lens as

$$
V=\frac{k}{\mu} \frac{P_{R}-P_{f}}{z_{h}}
$$

where $k$ is the permeability of the soil, $\mu$ is the dynamic viscosity of water, and $z_{h}$ is the distance between the ice lens and the reservoir of warm water. $P_{f}$ is the pressure of the water directly below the warmest lens. Capillary theory assumes local equilibrium at the ice lens-soil boundary so that $P_{f}$ is given by the Clapeyron equation (2), and with this assumption equation (7) determines $V$. However at rates typical of frost-heave experiments the equation tends to overpredict measured values of $V$ by as much as several orders of magnitude $[15,16,33,32]$.

3) No mechanism for initiation of new lenses. A significant feature of frost heave is the sudden appearance of a new lens a finite distance below a previously growing lens, leading to a rhythmic banding structure such as shown schematically in figure 2(c). No 
plausible mechanism within the capillary theory was offered to explain why ice lenses form such discrete bands [34].

In Section 4 we review experimental and theoretical work demonstrating that these obstacles to the capillary theory have been largely resolved, leading to an improved capillary model. However, the difficulties seemed insurmountable in the 1970s and led to the development of a radically different approach - the frozen-fringe model of frost heave.

\section{Frozen fringe models}

The apparent failure of the capillary theory led some researchers [35, 36] to propose that frost heave can continue to occur at ice-lens temperatures below $T_{p}$, i.e. after ice has formed a frozen fringe by growing into the pores of the soil (figure 3 ). This assumption was supported by experiments and modelling that demonstrated the existance of premelted films at temperatures below $T_{p}$, thus potentially allowing slow transport of water through the partially frozen region of the soil $[37,19]$. The driving force causing flow in the fringe, while initially uncertain, was eventually shown to be caused by thermomolecular pressure gradients in the premelted films [38, 39]. An elegant demonstration of this process was provided by Wilen and Dash [40], who developed a simple experimental analogue of a frozen fringe using a capillary, and measured the flow in the premelted films. Theoretical modelling using lubrication theory yielded very good agreement with the experimental results $[41,42]$.

Harlan [36] was the first to propose equations for heat and matter flow in a frozen fringe. His conservation of energy equation accounting for the phase change of unfrozen water to ice took the form

$$
\rho_{s} c_{p s} \frac{\partial T}{\partial t}=\frac{\partial}{\partial z}\left(k_{s} \frac{\partial T}{\partial z}\right)+\rho_{i} L_{f} \frac{\partial \phi_{i}}{\partial t}
$$

where $\rho_{s}, c_{p s}$ and $k_{s}$ are the density, specific heat capacity and thermal conductivity of the partially frozen soil, and $\phi_{i}$ is the volume fraction of ice in the pore space, related to the volume fraction of unfrozen water $\phi_{w}$ and soil particles $\phi_{p}$ by the identity $\phi_{i}+\phi_{w}+\phi_{p}=1$. In physical terms, this energy equation can be thought of as a diffusion equation for heat in the frozen fringe, with an added source term due to latent heat release as the liquid fraction freezes. To model mass transport Harlan [36] assumed Darcy's law applies in 


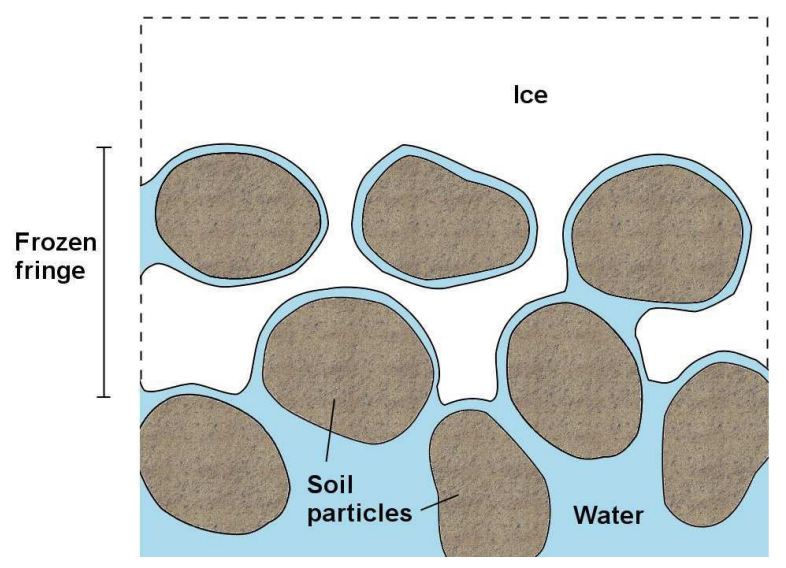

Figure 3: Schematic diagram of a freezing soil with a frozen fringe

the fringe, with an effective permeability that is a function of the water fraction $\phi_{w}$. Although Harlan's equations did not permit the formation of discrete lenses, his theory provided a structure upon which subsequent researchers were able to propose conditions for new lenses.

The frozen-fringe hypothesis provided a potential resolution to the problems described in Section 2.3. Firstly, the suggestion that an ice lens can continue to grow after the ice invades the pore space means that there is no longer the constraint (6) on the maximum frost-heave pressure, allowing for larger values of $P_{m}$. Secondly, partial blocking of the pores of the soil in the frozen fringe causes slower flow of water towards a growing lens, and thus slower heave rates, potentially resolving the second difficulty with capillary theory. Finally, the existence of ice ahead of the growing lens provides nuclei from which new lenses can form and allows mechanisms to be advanced for the initiation of new lenses. For example, Miller [35, 34, 43, 44] built on the Harlan model by proposing an equation

$$
\sigma_{n}=\sigma+(1-\chi) P_{i}+\chi P_{w}
$$

to determine the effective stress $\sigma_{n}$ acting on particles in the frozen fringe. Here $\sigma=-P_{o}$ is the total stress and $\chi$ is a semi-empirical stress partition function that describes how the overlying weight is distributed between the pore ice and pore water in the frozen fringe. This function was introduced based on a similar stress partition function developed for unsaturated soil mechanics $[45,34]$. Using Harlan's model to determine the temperature and fluid pressure in the fringe, Miller [34, 43] estimated the ice pressure using the Clapeyron equation and used this to keep track of $\sigma_{n}$. He found that, under certain 
conditions, the effective stress between soil particles becomes positive - so the particles in the soil can in principle separate - and proposed that a new ice lens would form at this point.

Miller's theory, which he referred to as secondary frost heaving (with capillary theory referred to as primary heaving), was the first to yield quantitative predictions of lens spacings. The frozen fringe concept has formed the basis of a large number of subsequent theories $[46,47,48,49,50,51,52,53,54,55,56,57,58,59,60,61,62,63,39,64]$. These works propose different physical explanations for the flow of premelted water in the frozen fringe [39], as well as various mechanisms to characterize the stress state leading to different ice-lens initiation criteria $[46,54,56,39]$. There has also been substantial work to provide simpler numerical solutions of the complex transport equations in the frozen fringe. For example, Gilpin [46] proposed a somewhat simpler model than Miller's, in which new ice lenses form when the calculated ice pressure in the frozen fringe reaches a critical separation pressure. Fowler [54] simplified the mathematics of the Miller model by assuming the fringe thickness is asymptotically small. His theory allows for two dimensional effects, potentially explaining differential frost heave and patterned ground $[57,65]$. Rempel et al. [39, 66] generalized models of flow in premelted films [41, 42] to derive an expression for fluid transport in the frozen fringe. By performing a force balance on particles in the fringe the model distinguishes parameter regimes for the growth of a single lens, growth of multiple lenses to form a banded sequence, and freezing of the pore space with no ice lenses.

As an example of the predictions of frozen-fringe models, figure 4 shows how different frost-heave behaviours occur depending on the applied freezing conditions for representative soil parameters [39]. The diagram plots a nondimensional freezing velocity versus a nondimensional overburden pressure, with $G$ being the temperature gradient in the sample. There are three main regimes that occur as the soil initially freezes: Firstly, in the light grey region (at low $V$ and $P_{o}$ ) a single ice lens will grow in a stable manner, pushing all the soil particles ahead of it. Secondly, at higher freezing speeds in the white region, periodic ice lenses form. Thirdly, when $P_{o}$ is sufficiently large (dark grey region), no heave can occur and so no ice lenses form. Rempel et al. [39] also noted that hysteresis is predicted near the regime boundaries (dashed lines). For instance, if the soil is initially frozen in the periodic lens regime, and the freezing rate is dropped, the soil will not revert to the steady lens behaviour until the conditions move below the bottom 


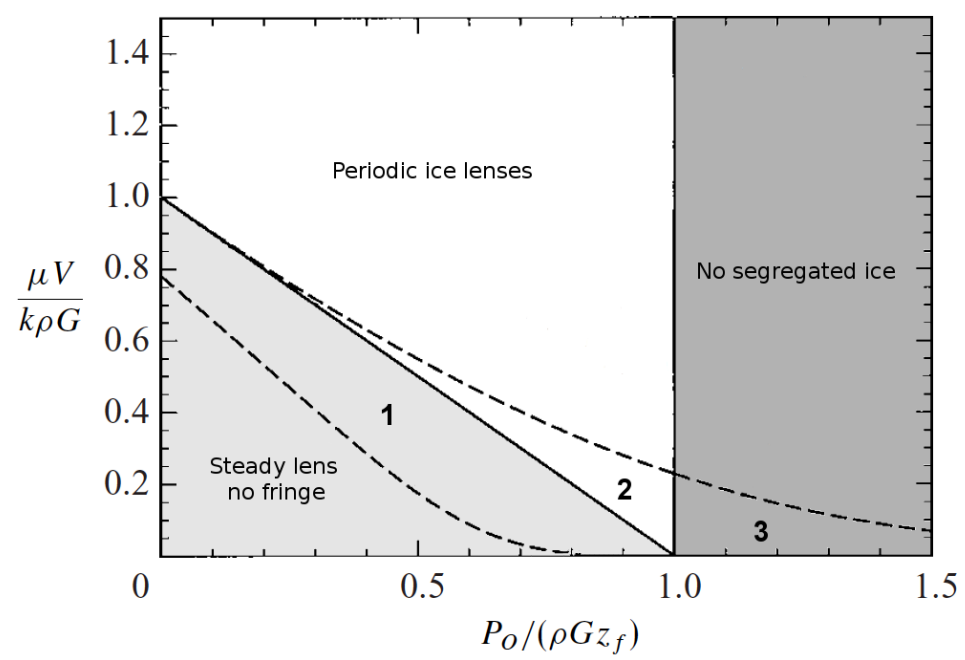

Figure 4: Regime diagram for soil freezing behaviour at different freezing speeds and overburden pressures, modified from Rempel et al. [39]. The behaviour in the different regimes is described in the text.

dashed line. Thus the behaviour in regimes 1 and 2 can be either a steady single lens or periodic ice lensing. Similarly in regime 3, there can either be no segregated ice, or a steady single lens.

Results such as those in figure 4 show that frozen fringe models can replicate many of the qualitative changes in behaviour that are seen in experiments. Frozen fringe models have also had some success at making quantitative predictions of heave rates $[46,58$, 67], though detailed comparisons with experiment have proven challenging [68]. Further details regarding the capillary and frozen-fringe models can be found in review articles by Rempel [69], Dash et al. [19], Black [68], Smith [70] and O’Neill [71].

\subsection{Does the frozen fringe exist?}

A key question distinguishing the capillary and secondary heave models is whether ice lenses form within a frozen fringe, or whether they always grow without entering the pore space. Despite a substantial amount of experimental work, this has proven difficult to answer. The pore size in typical frost-heaving soils is on the order of $1 \mu \mathrm{m}$ in diameter, and so pore-scale effects are difficult to probe directly. Furthermore, the indices of refraction of water and ice are very close to each other which means that it is not possible to distinguish optically when pore ice appears, unlike the process of soil desaturation [72]. 
Thus many experiments have resorted to indirect estimation of when a frozen fringe has occurred, for instance by estimating when the temperature drops below $T_{p}[73,74]$.

That ice lenses can form in a frozen fringe is perhaps best demonstrated by their appearance in rocks. In this case the migration of freezing water into large pores or microcracks of the rock pushes open the cracks, leading to fracturing of the rock and the formation of new lenses $[75,76]$. It has been shown that the pressures that act to force open new cracks are only sufficiently large to fracture the rock when the temperature drops well below the pore freezing temperature of the rock [74]. In this case it seems likely that water is drawn through a frozen-fringe-like region of rock to feed the growing ice lenses.

Other evidence for frozen fringes are not so clear cut. For instance, one of the main experiments supporting the frozen-fringe hypothesis in silty soils was published by Loch \& Kay [73]. They measured the temperature of ice-lens formation in New Hampshire silt and found that it was colder than their estimation of the pore freezing temperature $T_{p}$. This appears to be evidence supporting the existence of a frozen fringe. However, Loch \& Kay did not take account of the polydispersity of the soil, known to have significant impacts on $T_{p}[11,30]$. If we use the expression of Everett [11] for the ice-entry temperature of a polydisperse soil to recalculate $T_{p}$ for Loch \& Kay's experiment then the results are less certain (see Section 4).

To further complicate matters there is an increasing amount of experimental data showing that in soils composed of clay and fine silt sized particles, ice lenses can indeed form without a frozen fringe. For example, Beskow [7] stressed repeatedly that in clays and fine silts the soil between the warmest ice lenses is unfrozen:

The very important fact that in an ice-banded frozen soil with a moderately low temperature, the soil between the ice bands is fully plastic and soft, therefore unfrozen, has not been considered... In fine clays, cooled only a few degrees below freezing, all the pore water is unfrozen, leaving the clay between the ice layers plastic and soft...

he goes on to say

Near the frost line (where the temperature is only slightly below $0{ }^{\circ} \mathrm{C}$ ) the soil between the ice layers is unfrozen and soft even in silty soils.... [In clays] not only near the frost line, but also higher up, the soil is just as soft and plastic 
as it is under this level. Thus in clays at moderately low temperatures, the soil itself between the ice layers is soft and unfrozen.

Brown [77] placed blocks of saturated soil on a thin layer of ice and then slowly reduced the temperature of the system. He found that, during sufficiently slow freezing, water from the soil would flow to the ice, causing it to thicken while the soil consolidated. At a critical temperature the soil block stopped shrinking and upon examination was solid and frozen. Brown concluded that ice had entered the pores and defined this critical temperature as the pore freezing temperature $T_{p}$. The value for $T_{p}$ was later confirmed via differential scanning calorimetry experiments [78]. Importantly, Brown [77] noticed that if the temperature was reduced too quickly, ice lenses formed in the interior of the block of soil. These ice lenses always formed at temperatures warmer than $T_{p}$, consistent with the capillary theory and contradicting the frozen-fringe hypothesis (which requires ice lenses to form at temperatures colder than $T_{p}$ ).

Akagawa [79] measured the thermal conductivity of frozen soil, anticipating that the thermal properties of frozen soil would be distinct from those of unfrozen soil. As expected Akagawa [79] found that the thermal conductivity of regions of soil containing ice lenses was higher than that of the unfrozen soil. In contrast, in the region between the warmest ice lens and the $0{ }^{\circ} \mathrm{C}$ isotherm (where the frozen fringe was expected to be located) the measured thermal conductivity was indistinguishable from that of the unfrozen soil [79].

Takeda and Okamura [80] and Watanabe et al. [81] used light microscopy to examine under high magnification the soil adjacent to the warmest ice lens in freezing samples of Kanto loam and Fujinomori clay. They found no evidence of ice in the frozen fringe region, and no significant structural changes that might be expected if ice were forming in the pores of the soil. In one experiment, Takeda and Okamura [80] followed a large fluid-filled pore as it migrated into the frozen fringe region. If the fringe contained ice, it was expected that water in the large pore would freeze. However, the water remained unfrozen until the pore was broken by a crack-shaped ice lens.

Finally, Watanabe and Mizoguchi [82] devised an improved version of the Loch and Kay [73] experiment. They developed a novel Raman-spectroscopy technique capable of detecting pore ice. In a setup similar to Loch and Kay's, and using a soil composed of fine, silt-sized particles (10 $\mu \mathrm{m}$ diameter), Watanabe and Mizoguchi [82] found that no pore ice was present in the soil ahead of the growing ice lens. As a result they concluded that frozen fringe theories are not applicable to their system. 
As a result of conflicting experimental results, it does not seem possible at the present time to conclusively say whether or not frozen fringes exist. Indeed it is likely that a frozen fringe may be present in some systems and not in others, with its presence depending upon the soil material and the rate of freezing. Importantly though, on the basis of the evidence above, we can conclude that in some systems a sequence of ice lenses can form without the presence of a frozen fringe. This suggests that there must be a mechanism by which new ice lenses can form within the framework of the capillary theory. In the next section we describe recent work that has revived the capillary theory by proposing new mechanisms for the initiation of ice lenses and potentially resolving the objections raised in Section 2.3.

\section{Revised capillary theory}

With recent experimental work seeming to conclusively demonstrate ice-lens formation without a frozen fringe in some systems, frost heave researchers have been led to readdress the problems with capillary theory. Here we show that the major objections to the capillary model listed in Section 2.3 can be resolved via a combination of early neglected explanations and more recent results.

1) $P_{m}$ predictions deviated from experiments in polydisperse soils. As discussed in Section 2.3, the capillary theory accurately predicts the maximum heaving pressures in monodisperse soils, but gives smaller pressures than observed in tests on soils with a broad particle-size distribution. It can be shown, however, that an error is introduced if the effective pore size is estimated based on air-entry measurements. Studies have shown that air entry values are determined by the largest pore sizes in a sample, which in turn are determined by the largest particle sizes in the sample [83]. As an example, in the experiments of Loch and Kay [73] discussed in Section 3.1, a pore size of $8 \mu \mathrm{m}$ was obtained from air-entry measurements, corresponding to the largest $10 \%$ of particles in their sample.

In contrast to the air-entry process, when ice grows next to a soil surface a sorting process occurs, reducing the effective pore size of the soil. That is, the largest particles in a sample tend to be engulfed by an ice interface while smaller particles are pushed ahead $[84,85,86]$. Thus a boundary layer of relatively small particles will tend to form against a growing ice lens, with the inter-particle pore size being significantly reduced. 
This means that the pore size at ice entry can be significantly smaller than the pore size at air entry.

If we accept assertions that the ice-entry pressure is determined by the smallest particles in a soil due to the mechanism above [11,30], the apparent contradictions with capillary theory appear to be resolvable. For example, Sutherland and Gaskin [30] show that good agreement with equation (3) is obtained if Everett's [11] proposal that $T_{p}$ is determined by the smallest $10 \%$ of particles is used to determine maximum pressures. Experiments capable of monitoring particle size and rearrangements at the surface of a growing ice lens will help to further clarify this issue.

2) Failure of Clapeyron equation outside equilibrium. Even in soils composed of uniform particles, the Clapeyron equation (1) was observed to break down at significant freezing rates (as opposed to equilibrium measurements taken after ice lenses stopped growing, when the equation works well) $[15,16,33]$. A first explanation for this was proposed by Jackson et al. [10] who demonstrated that viscous flow in premelted films between an ice lens and soil particles becomes important at finite freezing rates. Similar ideas were presented by several subsequent authors $[18,87,88,89,90,91,92,32]$. As demonstrated by Style and Peppin [32], accounting for the viscous resistance to flow in the films shows that the Darcy pore pressure at the ice lens $P_{f}$ (c.f. figure 2(c)) is given by a generalized Clapeyron equation containing a kinetic term:

$$
P_{f}=P_{o}-\frac{\rho_{w} L_{f}}{T_{m}}\left(T_{m}-T\right)+V f\left(T_{m}-T\right),
$$

where $V$ is the growth rate of the ice lens and $f$ is a function of temperature that can be measured or calculated from the geometry of the soil particles. The Clapeyron equation (2) is recovered when the growth rate is small, however the additional term in equation (10) causes the flow rate to the growing ice lens to be significantly reduced in typical freezing scenarios. Using (10) with Darcy's law (7), predicted flow rates can be matched with experimental measurements [32].

Equation (10) also quantifies the dependence of frost heave on particle size. For soils made up of larger particles, the viscous resistance effect results in a large reduction in flow rate, as typically $f\left(T_{m}-T\right)$ is proportional to the square of the particle size [32]. On the other hand, for soils consisting of smaller particles the flow rate is reduced because of the low permeability of the soil. Thus there is a maximum heave rate for soils composed of intermediate-sized particles. This is seen in experiments, where frost-susceptibility is 
known to be greatest for medium-grained soils such as silts [7]. Equation (10) can in principle predict the optimum particle size for soil of a given material [32].

3) Mechanism for initiation of new lenses. As discussed in Section 2.3, a major drawback of the capillary theory was its failure to explain how ice lenses form in discrete bands. Beskow [7] and Martin [93] suggested that the region in front of a growing ice lens would become progressively supercooled, allowing a new ice lens to nucleate in a flaw or large pore. Beskow [7] further noted the resemblance of ice lens growth to a fracture process, and hypothesized that supercooling ahead of a growing ice lens may provide a source of energy for a new lens to nucleate. However, these ideas were not quantified or explored further. Nevertheless, recent work has shown that the basic ideas have merit, with two main potential mechanisms for fringe-free ice-lens formation having been proposed.

\subsection{Fringe-free models of intermittent lensing}

\subsubsection{Engulfment model}

Mutou et al. [94] performed a series of experiments where they froze a dilute suspension of soil particles in a cell. They did this by imposing a fixed temperature gradient on the cell, and then pulling the cell at a fixed rate, so that freezing occurred at a constant speed. For pulling speeds in excess of a critical velocity $V_{c}$ all the particles were engulfed by the ice front, while for speeds below $V_{c}$ the particles were pushed ahead by the ice - just as single particles are rejected ahead of a growing ice lens at low freezing speeds [85, 86]. At speeds just below $V_{c}$, Mutou et al. [94] observed that a layer of particles would build up against the ice interface and then suddenly become engulfed when the layer reached a certain thickness. Repetition of this process yielded a banded structure which partially resembled a sequence of ice lenses. Watanabe et al. [95] explained the engulfment of the layer as owing to the viscous drag of the particle layer (in addition to the drag of the premelted films at the ice-particle layer interface) which reduces the effective critical velocity required for engulfment. They hypothesized that a similar process occurs during ice lens formation in soils [95]. That is, a compacted region of particles builds up against the warm face of a growing ice lens. Once the compacted region reaches a certain critical thickness the ice interface engulfs the compacted layer and a new lens forms at a less consolidated region of soil. A similar explanation was proposed by Jackson et al. [10] 
and Zhu et al. [96].

The engulfment model is a viable mechanism for the formation of ice bands. Whether such bands are the same thing as ice lenses is an open question, with recent experiments suggesting bands form at much higher freezing velocities than ice lenses [97, 98]. In addition the model of Watanabe et al. [95] requires the soil between ice lenses to be frozen and hence cannot explain some observations of clays and fine silts that show the soil between the warmest ice lenses to be unfrozen [7, 77]. A useful test of the theory would therefore be to use a technique such as that of Watanabe \& Mizoguchi's [82] to search for pore ice in the soil on the cold side of a growing ice lens.

\subsubsection{Geometrical supercooling model}

In order to gain insight into observations of fringe-free ice lens formation, Peppin et al. [99] developed a Stefan model of the growth of a single ice lens adjacent to a saturated soil composed of rigid spherical particles. The model finds that the soil adjacent to an ice lens consolidates and at fast freezing rates can become constitutionally supercooled. This constitutional supercooling is entirely analogous to the constitutional supercooling that is found in solidifying alloys and freezing aqueous solutions [100]. Later work demonstrated that, in non-cohesive soils and colloidal suspensions, when constitutional supercooling occurs the ice/soil interface is morphologically unstable [101, 102]. The instability results in dendritic ice structures which grow rapidly into the freezing suspension [101]. This model provides a thermodynamic explanation for the presence of supercooling and segregated ice in otherwise unfrozen soil, though gives little information on the structure and orientation of the ice. Extensions of this work led to a "mushy layer" model of frost heave [103] analogous to mushy layer models of dendritic ice in alloys [104]. Similar mixed-phase models were proposed by Arakawa [105] and Chalmers and Jackson [106]. Peppin et al. [103] and Chalmers and Jackson [106] found using this model that the rate of heave is independent of the rate of soil freezing, in agreement with experimental results of Beskow [7], Ueda and Penner [107] and Watanabe [33].

Style et al. [14] extended the constitutional supercooling concept to cohesive soils that may or may not contain pore ice and referred to the phenomenon in general as geometrical supercooling. They extended the single ice-lens model [99] to look at the anisotropic stress state that develops in a cohesive soil next to a growing ice lens. Motivated by observations which showed that the opening of a new ice lens appears to be a fracture process $[7,99]$, 
they considered the growth of an ice-filled flaw in the supercooled region ahead of a growing lens. When the ice pressure in the flaw reaches a critical pressure $P_{i}=P_{o}+\sigma_{t}$ it overcomes the tensile strength of the soil $\sigma_{t}$ and the overlying weight $P_{o}$. This allows the flaw to crack open across the soil forming a new lens. The critical condition can be rewritten as

$$
T_{c l}-T=\Delta T_{c}
$$

where $T_{c l}$ is the Clapeyron temperature, that is, the temperature at which a new ice lens can exist stably at equilibrium, and $\Delta T_{c}=T_{m} \sigma_{t} /\left(\rho_{w} L_{f}\right)$. For a soil with pore pressure profile $P_{w}(z, t)$ and overburden $P_{o}$, the Clapeyron temperature is determined from (1) as

$$
T_{c l}(z, t)=T_{m}\left(1-\frac{P_{o}-P_{w}(z, t)}{\rho_{w} L_{f}}\right) .
$$

When the gradient in Clapeyron temperature at the ice lens surface is larger than the temperature gradient, ie when

$$
\frac{\partial T_{c l}}{\partial z}>\frac{\partial T}{\partial z}
$$

the soil ahead of the lens is geometrically supercooled. A representative profile of $T$ and $T_{c l}$ is shown schematically in figure 5 . The maximum geometrical supercooling occurs at a finite distance ahead of the growing lens, and when this maximum reaches $\Delta T_{c}$, a new ice lens can form. As shown in section 4, the temperature at the surface of the ice lens is given by equation (10) as $T=T_{c l}-V / \kappa_{u}$, where $\kappa_{u}=\rho_{w} L_{f} /\left[T_{m} f\left(T_{m}-T\right)\right]$ is a kinetic supercooling coefficient. Figure 5 illustrates the case considered by Style et al. [14] when $\kappa_{u}$ is large and the kinetic term is small.

Style et al. [14] showed that geometrical supercooling will occur when there is a significant drop in the permeability of the soil directly ahead of the growing ice lens. They suggested three possible causes: the formation of a frozen fringe, desaturation of the soil, and compaction of a compressible soil. Each of these is known to occur, and will certainly provide enough supercooling to allow periodic ice lenses to grow [14]. Importantly the latter two mechanisms do not require a frozen fringe to be present.

An obvious difficulty is how an ice-filled crack appears ahead of an ice lens if there is no frozen fringe. Style et al. [14] conclude, as did Scherer [108], that spontaneous nucleation of ice in pores is not likely. Instead they suggest that new lenses can nucleate from the side of ice-filled shrinkage cracks that are known to extend some distance ahead of the warmest ice lens $[7,109,110]$. This is shown schematically in figure $6(a)$, while figure 6(b) shows shrinkage cracks protruding ahead of an ice lens in a typical freezing soil 


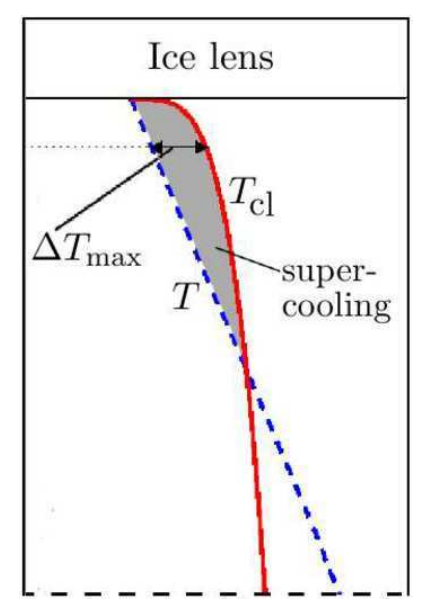

Figure 5: Schematic diagram showing geometrical supercooling in a freezing soil. A maximum in the supercooling exists at a finite distance ahead of the growing ice lens. A new lens will form at this point when the supercooling reaches the $\Delta T_{c}$.

[110]. Ice lens formation in this manner would result in ladder-like patterns of segregated ice, and this is indeed seen in many experiments $[7,109,101,110]$, with a typical example in figure 7. Alternatively new lenses may nucleate off pre-existing lenses as cracks which curve down to form a new lens, resulting in a different morphology which is also seen in frost heave experiments $[5,6]$.

This theory shows very good qualitative agreement with experimental observations. Quantitatively, the model agrees with measurements of the temperature at which new ice lenses form in two experimental systems [14], and work is currently ongoing to test predictions of lens spacings and lens thicknesses against additional experiments. Further application of the principles and procedures of fracture mechanics may be useful in determining the key parameters are that control ice-lens patterns during freezing.

\section{Discussion}

As will have become apparent, there are still many questions that must be answered in order to give a complete picture of the physics of frost heave. Several experimental issues that present themselves are:

- Under what conditions does frozen-fringe/fringe-free formation of periodic lenses occur? 

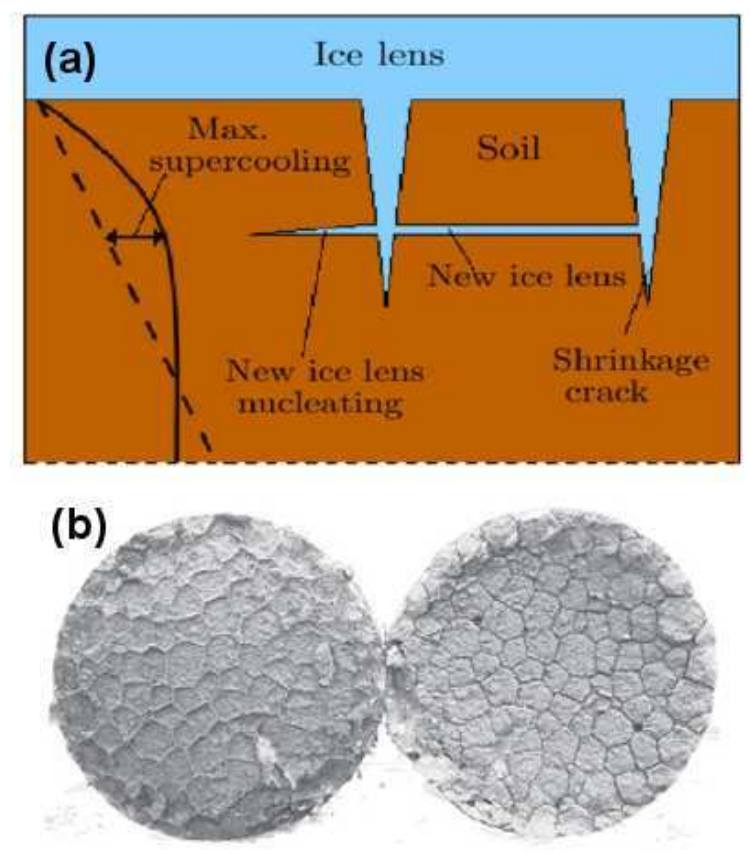

Figure 6: (a) Schematic diagram showing how new ice lenses can nucleate off the side of shrinkage cracks that grow into the soil ahead of an existing ice lens. (b) A freezing block of Devon silt that is broken open at the freezing front [110]. The left hand block is the upper, frozen soil. The right hand block is the warmer, unfrozen soil. A polygonal network of shrinkage cracks can clearly be seen extending out of the frozen material. The sample diameter is about $100 \mathrm{~mm}$.

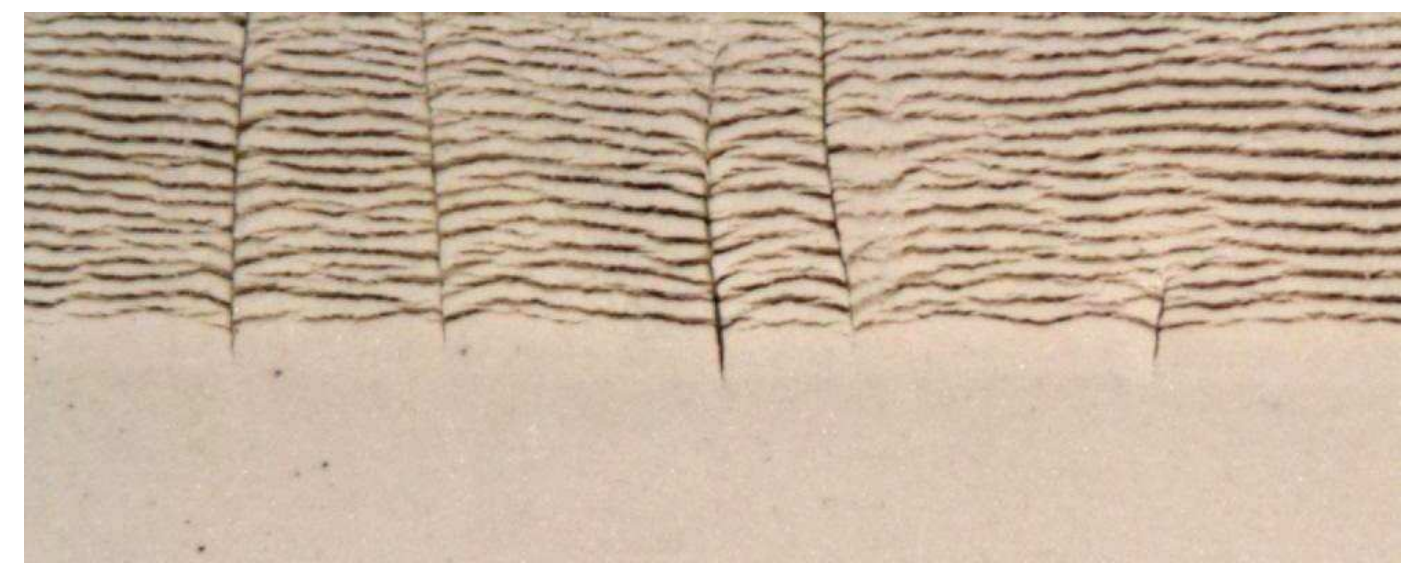

Figure 7: Ice lenses forming in a freezing sample of kaolinite. The clay is frozen in the directional solidification of Peppin et al. [102]. Image height is approximately $2 \mathrm{~cm}$. Vertical shrinkage cracks can clearly be seen, giving the ladder-like structure discussed in the text. 
- Can the same type of soil exhibit periodic lensing with and without a frozen fringe?

- If both frozen-fringe and fringe-free lensing occur, is one mode more dangerous than the other? We might expect fringe-free heave rates to be faster due to the lack of pore-blocking by ice.

- Can the engulfment model of ice lenses be verified by looking for pore ice behind newly formed ice lenses?

At the same time, there is still a substantial amount of theoretical work to be done. One of the key reasons for understanding frost heave is to be able to understand soil movement for geophysical and engineering applications, and so it is important to be able to extend the models above into three dimensions. Both theoretical and experimental work are also needed to explore further aspects of frost heave that have not been captured above. For instance

- How is frost heave affected by the presence of solutes in the pore water [111], soil cohesiveness, polydispersity [11,30] and unsaturated conditions [112]?

- How do ice-filled cracks propagate through a soil? What controls their horizontal growth rate? Can they be modelled with unfrozen soil parameters?

- Can frost heave models be extended to two and three dimensions and used to explain the formation of patterned ground [65]? Can such models of patterned ground be used to give insight on past climate conditions $[113,1]$ ?

The fact that so many questions remain demonstrates that, despite its long history, there is still much work to be done to understand frost heave and its effects. However, with the recent progress made using new experimental and theoretical techniques, this is certainly an exciting time to be involved in the field. We hope that this review serves to stimulate new research that can advance the field, and unlock many of the puzzles that remain.

\section{Conclusion}

In this review we have discussed the various mathematical models that have been proposed to explain frost heave and the growth of ice lenses in freezing soils. While early 
theories based on the capillary model captured many of the essential features and showed good agreement with experiment, deficiencies of the model including its underprediction of frost heave pressures and its failure to explain the rhythmic banding of ice lenses led many researchers to abandon it in favour of a fundamentally distinct secondary frost heave model. The secondary heave model allows ice lenses to form within a partially frozen fringe, in contrast to the capillary theory which only permits ice lenses to grow at the freezing front. Experimental work is divided over the issue: in some systems a frozen fringe appears to be present, while in others it is absent. The latter observations have motivated a revisit of the capillary theory, and recent work shows that its previous deficiencies have been substantially resolved, albeit with the theory in a significantly revised form. The particle-engulfment and the geometrical supercooling models are summarized yielding new mechanisms for the periodic formation of ice lenses that are consistent with capillary theory. Some open problems are also discussed. It is hoped the review will stimulate experimental and theoretical developments leading to further insight into an intriguing geophysical phenomenon.

\section{Acknowledgements}

This publication is based on work supported by Award No. KUK-C1-013-04, made by King Abdullah University of Science and Technology (KAUST). RWS is funded by a Yale University Bateman Interdepartmental Postdoctoral Fellowship.

\section{References}

[1] C. Gallagher, M.R. Balme, S.J. Conway, and P.M. Grindrod. Sorted clastic stripes, lobes and associated gullies in high-latitude craters on mars: Landforms indicative of very recent, polycyclic ground-ice thaw and liquid flows. Icarus, 211:458-471, 2011.

[2] A. DiMillio. A Quarter Century of Geotechnical Research. Federal Highway Administration, Washington, DC, 1999.

[3] A. C. Palmer and P. J. Williams. Frost heave and pipeline upheaval buckling. Canadian Geotechnical Journal, 40:1033-1038, 2003.

[4] S. Taber. The growth of cyrstals under external pressure. American Journal of Science, $37: 532-556,1916$. 
[5] S. Taber. Frost heaving. J. Geol., 34:428, 1929.

[6] S. Taber. The mechanics of frost heaving. Journal of Geology, 38:303-317, 1930.

[7] G. Beskow. The Swedish Geological Society, C, no. 375, Year Book no. 3. Technological Institute, Northwestern University, 1935. Reprinted in Historical Perspectives in Frost Heave Research (P. B. Black and M. J. Hardenberg, eds.) CRREL Special Report No. 91-23, pp 37-157, 1991.

[8] L. W. Gold. A possible force mechanism associated with the freezing of water in porous materials. Highway Research Board Bulletin, 168:65-72, 1957.

[9] K. Jackson and B. Chalmers. Freezing liquids in porous media with reference to frost heaving in soils. Journal of Applied Physics, 29:1178-1181, 1958.

[10] K. A. Jackson, D. R. Uhlmann, and B. Chalmers. Frost heave in soils. Journal of Applied Physics, 37:848-852, 1966.

[11] D. H. Everett. The thermodynamics of frost damage to porous solids. Transactions of the Faraday Society, 57:1541-1551, 1961.

[12] I. Prigogine and R. Defay. Chemical Thermodynamics. Longmans Green and Co., London, 1954.

[13] P. B. Black. Applications of the Clapeyron equation to ice and water in porous media, pages 1-7. CRREL Special Report No. 95-6. 1995.

[14] R. W. Style, S. S. L. Peppin, A. C. F. Cocks, and J. S. Wettlaufer. Ice lens formation and geometrical supercooling in soils and other colloidal materials. Physical Review E, 84:041402, 2011.

[15] F. J. Radd and D. H. Oertle. Experimental pressure studies of frost heave mechanisms and the growth-fusion behaviour of ice. In 2nd International Conference on Permafrost, pages 122-129, Yakutsk, 1960.

[16] M. B. G. M. Biermans, K. M. Dijkema, and D. A. De Vries. Water movement in porous media towards an ice front. Journal of Hydrology, 37:137-148, 1978.

[17] H. Ozawa and S. Kinosita. Segregated ice growth on a microporous filter. Journal of Colloid and Interface Science, 132:113-124, 1989.

[18] B. V. Derjaguin and N. V. Churaev. The theory of frost heaving. Journal of Colloid and Interface Science, 67:391-396, 1978. 
[19] J. G. Dash, A. W. Rempel, and J. S. Wettlaufer. The physics of premelted ice and its geophysical consequences. Rev. Mod. Phys., 78:695-741, 2006.

[20] R. Defay and I. Prigogine. Surface Tension and Adsorption. Longmans, London, 1966.

[21] E. Penner. The mechanism of frost heaving in soils. Highway Research Board Bulletin, $225: 1-13,1959$.

[22] E. Penner. Pressures developed in a porous granular system as a result of ice segregation. Highway Research Board Special Report, 40:191-199, 1959.

[23] R. D. Miller, J. H. Baker, and J. H. Kolaian. Particle size, overburden pressure, pore water pressure and freezing temperature of ice lenses in soils. In 7th International Congress of Soil Scientists, Transactions, volume 1, pages 122-129, 1960.

[24] E. Penner. Pressures developed during the unidirectional freezing of water-saturated porous materials. In Proceedings of the International Conference on Low Temperature Science, volume 1, pages 1401-1412, Sapporo, Japan, 1967.

[25] E. Penner. Frost heaving pressures in particulate materials. In Organisation for Ecomomic Co-Operation and Development Symposium on Frost Action on Roads, volume 1, pages 379-385, Paris, France, 1973.

[26] M. Vignes and K. M. Dijkema. A model for the freezing of water in a dispersed medium. Journal of Colloid and Interface Science, 49:165-172, 1974.

[27] E. Penner and L. E. Goodrich. Location of segregated ice in frost-susceptible soil. Engineering Geology, 18:231-244, 1981.

[28] E. Penner. Heaving pressure is soils during unidirectional freezing. Canadian Geotechnical Journal, 4:398-408, 1967.

[29] J.P.G. Loch and R. D. Miller. Tests of the concept of secondary heaving. Soil Science Society of America Proceedings, 39:1036-1041, 1975.

[30] H. B. Sutherland and P. N. Gaskin. Pore water and heaving pressures developed in partially frozen soils. In 2nd International Conference on Permafrost, volume 1, pages 409-419, Yakutsk, 1973.

[31] M. K. Hubbert. The Theory of Ground Water Motion. Hafner Publishing Co., NY, 1969.

[32] R. W. Style and S. S. L. Peppin. The kinetics of ice-lens growth in porous media. Journal of Fluid Mechanics, 692:482-498, 2012. 
[33] K. Watanabe. Relationship between growth rate and supercooling in the formation of ice lenses in a glass powder. J. Cryst. Growth, 237:2194-2198, 2002.

[34] R. D. Miller. Lens initiation in secondary heaving. In Proceedings of the International Symposium on Frost Action in Soils, volume 2, pages 68-74, 1977.

[35] R.D. Miller. Freezing and heaving of saturated and unsaturated soils. Highway Research Record, 393:1-11, 1972.

[36] R. L. Harlan. Analysis of coupled heat-fluid transport in partially frozen soil. Water Resources Research, 9:1314-1323, 1973.

[37] J. W. Cahn, J. G. Dash, and H.-Y. Fu. Theory of ice premelting in monosized powders. J. Cryst. Growth, 123:101-108, 1992.

[38] J. G. Dash. Thermomolecular pressure in surface melting: a motivation for frost heave. Science, 246:1591, 1989.

[39] A. W. Rempel, J. S. Wettlaufer, and M. G. Worster. Premelting dynamics in a continuum model of frost heave. J. Fluid Mech., 498:227-244, 2004.

[40] L. A. Wilen and J. G. Dash. Frost heave dynamics at a single crystal interface. Physical Review Letters, 74:5076-5079, 1995.

[41] J. W. Wettlaufer and M. G. Worster. The dynamics of premelted films: Frost heave at a capillary. Physical Review E, 51:4679-4689, 1995.

[42] J. S. Wettlaufer and M. G. Worster. Premelting dynamics. Ann. Rev. Fluid Mech., $38: 427-452,2006$.

[43] R. D. Miller. Frost heaving in non-colloidal soils. In Proceedings of the 3rd International Conference on Permafrost, volume 1, pages 708-713, 1978.

[44] K. O'Neill and R. D. Miller. Exploration of a rigid ice model of frost heave. Water Resources Research, 21:281-296, 1985.

[45] A. W. Bishop and G. E. Blight. Some aspects of effective stress in saturated and partially saturated soils. Geotechnique, 13:177-197, 1963.

[46] R. R. Gilpin. A model for the prediction of ice lensing and frost heave in soils. Water Resources Research, 16:918-930, 1980. 
[47] S. W. Hopke. A model for frost heave including overburden. Cold Regions Science and Technology, 3:177-183, 1980.

[48] J. M. Konrad and N. R. Morgenstern. A mechanistic theory of ice lens formation in fine-grained soils. Canadian Geotechnical Journal, 17:473-486, 1980.

[49] S. Takagi. The adsorption force theory of frost heaving. Cold Regions Science and Technology, 1:57-81, 1980.

[50] T. V. Hromadka, G. L. Guymon, and R. L. Berg. Some approaches to modeling phase change in freezing soils. Cold Regions Science and Technology, 4:137-145, 1981.

[51] M. Fremond, H. Ghidouche, and N. Point. Freezing of a porous medium with water supply coupled stefan problem. Journal of Mathematical Analysis and Applications, 108:371-402, 1985 .

[52] J. Walder and B. Hallet. A theoretical model of the fracture of rock during freezing. Geological Society of America Bulletin, 96:336-346, 1985.

[53] K. Horiguchi. An osmotic model for soil freezing. Cold Regions Science and Technology, $14: 13-22,1987$.

[54] A. Fowler. Secondary frost heaving in freezing soils. SIAM Journal on Applied Mathematics, 49:991-1008, 1989.

[55] Y. Nakano. Quasi-steady problems in freezing soils: I. analysis on the steady growth of an ice layer. Cold Regions Science and Technology, 17:207-226, 1990.

[56] J. F. Nixon. Discrete ice lens theory for frost heave in soils. Canadian Geotechnical Journal, 28:843-859, 1991.

[57] A. Fowler and W. B. Krantz. A generalized secondary frost heave model. SIAM Journal on Applied Mathematics, 54:1650-1675, 1993.

[58] J. M. Konrad and C. Duquennoi. A model for water transport and ice lensing in freezing soils. Water Resources Research, 29:3109-3124, 1993.

[59] R. L. Michalowski. A constitutive model of saturated soils for frost heave simulations. Cold Regions Science and Technology, 22:47-63, 1993.

[60] L. Bronfenbrener and E. Korin. Kinetic model for crystallization in porous media. International Journal of Heat and Mass Transfer, 40:1053-1059, 1997. 
[61] N. Li, F. Chen, B. Su, and G. Cheng. Theoretical frame of the saturated freezing soil. Cold Regions Science and Technology, 35:73-80, 2002.

[62] A. Y. Sheshukov and A. G. Egorov. Frozen barrier evolution in saturated porous media. Advances in Water Resources, 25:591-599, 2002.

[63] F. Talamucci. Freezing processes in porous media: Formation of ice lenses, swelling of the soil. Mathematical and Computer Modelling, 37:595-602, 2003.

[64] H. R. Thomas, P. Cleall, Y.-C. Li, C. Harris, and M. Kern-Leutschg. Modelling of cryogenic processes in permafrost and seasonally frozen soils. Geotechnique, 59:173-184, 2009.

[65] R. A. Peterson and W. B. Krantz. A mechanism for differential frost heave and its implications for patterned-ground formation. Journal of Glaciology, 49:69-80, 2003.

[66] A. W. Rempel. Formation of ice lenses and frost heave. J. Geophys. Res., 112:F02S21, 2007.

[67] L. Bronfenbrener and R. Bronfenbrener. Modeling frost heave in freezing soils. Cold Regions Science and Technology, 61:43-64, 2010.

[68] P. B. Black. Historical perspective of frost heave research, pages 1-7. CRREL Special Report No. 91-23. 1991.

[69] A. W. Rempel. Frost heave. Journal of Glaciology, 56:1122-1128, 2010.

[70] M. W. Smith. Models of soil freezing, pages 96-120. Field and Theory: Lectures in Geocryology. University of British Columbia Press, Vancouver, 1985.

[71] K. O'Neill and R. D. Miller. The physics of mathematical frost heave models: A review. Cold Regions Science and Technology, 6:275-291, 1983.

[72] L. Xu, S. Davies, A. B. Schofield, and D. A. Weitz. Dynamics of drying in 3d porous media. Physical Review Letters, 101:094502, 2008.

[73] J.P.G. Loch and B. D. Kay. Water redistribution in partially frozen, saturated silt under several temperature gradients and overburden loads. Soil Science Society of America Journal, 42:400-406, 1978.

[74] B. Hallet, J. S. Walder, and C. W. Stubbs. Weathering by segregation ice growth in microcracks at sustained sub- zero temperatures: Verification from an experimental study using acoustic emissions. Permafrost and Periglacial Processes, 2:283-300, 1991. 
[75] J. Walder and B. Hallet. A theoretical model of the fracture of rock during freezing. Geological Society of America Bulletin, 96:336-346, 1985.

[76] I. Vlahou and M. G. Worster. Ice growth in a spherical cavity of a porous medium. Journal of Glaciology, 56:271-277, 2010.

[77] S. C. Brown. Soil Freezing. PhD thesis, University of Reading, Reading, UK, 1984.

[78] S. C. Brown and D. Payne. Frost action in clay soils. ii. ice and water location and suction of unfrozen water in clays below $0{ }^{\circ}$ c. Journal of Soil Science, 41:547-561, 1990.

[79] S. Akagawa. Experimental study of frozen fringe characteristics. Cold Regions Science and Technology, 15:209-223, 1988.

[80] K. Takeda and A. Okamura. Microstructure of freezing front in freezing soils. Ground Freezing 97. Balkema, Rotterdam.

[81] K. Watanabe, M. Mizoguchi, T. Ishizaki, and M. Fukuda. Experimental study on microstructure near freezing front during soil freezing. Ground Freezing 97. Balkema, Rotterdam.

[82] K. Watanabe and M. Mizoguchi. Ice configuration near a growing ice lens in a freezing porous medium consisting of micro glass particles. J. Cryst. Growth, 213:135-140, 2000.

[83] D. Hillel. Introduction to Environmental Soil Physics. Elsevier, USA, 2004.

[84] A. E. Corte. Particle sorting by repeated freezing and thawing. Science, 142:499-501, 1963.

[85] D. R. Uhlmann, B. Chalmers, and K. A. Jackson. Interaction between particles and a solid/liquid interface. J. Appl. Phys., 35:2986-2992, 1964.

[86] A. W. Rempel and M. G. Worster. The interaction between a particle and an advancing solidification front. J. Cryst. Growth, 205:427-440, 1999.

[87] T. Forland and S. K. Ratkje. Irreversible thermodynamic treatment of frost heave. Engineering Geology, 18:225-229, 1981.

[88] B. V. Derjaguin and N. V. Churaev. Flow of nonfreezing water interlayers and frost heaving. Cold Regions Science and Technology, 12:57-66, 1986.

[89] T. Kuroda. Role of water layer at an ice surface in the kinetic processes of growth of ice crystals. Journal de Physique, 48:487-493, 1987. 
[90] T. Tsuneto. Remarks on frost heave. Journal of the Physical Society of Japan, 63:2231$2234,1994$.

[91] M. G. Worster and J. S. Wettlaufer. The Fluid Mechanics of Premelted Liquid Films, pages 339-351. Fluid Dynamics at Interfaces. Cambridge University Press, Cambridge, UK, 1999.

[92] A. W. Rempel. A theory for ice-till interactions and sediment entrainment beneath glaciers. J. Geophys. Res., 113:F01013, 2008.

[93] R. T. Martin. Rythmic ice banding in soil. Highway Research Board Bulletin, 218:11-23, 1959.

[94] Y. Mutou, K. Watanabe, M. Mizoguchi, and T. Ishizaki. Microscopic observation of ice lensing and frost heaves in glass beads. In Proceedings of the 7th International Conference on Permafrost, pages 783-787, Yellowknife, Canada, 1998.

[95] K. Watanabe, Y. Muto, and M. Mizoguchi. A model for the formation of ice lenses in an unconfined, water-saturated porous medium consisting of spherical particles. In Ground Freezing 2000. Frost Action in Soils: Proceedings of the 9th International Symposium, pages 55-60, Louvain-La-Neuve, Belgium, 2000.

[96] D.-M. Zhu, O. E. Vilches, J. G. Dash, B. Sing, and J. S. Wettlaufer. Frost heav in argon. Physical Review Letters, 85:4908-4911, 2000.

[97] S. Deville, E. Maire, G. Bernard-Granger, A. Lasalle, A. Bogner, C. Gauthier, J. Leloup, and C. Guizard. Metastable and unstable cellular solidification of colloidal suspensions. Nature Mat., 8:966-972, 2009.

[98] A. Lasalle, C. Guizard E. Maire, J. Adrien, and S. Deville. Particle redistribution and structural defect development during ice templating. Acta Materiala, page in press, 2012.

[99] S. S. L. Peppin, J. A. W. Elliott, and M. G. Worster. Solidification of colloidal suspensions. J. Fluid Mech., 554:147-166, 2006.

[100] S. H. Davis. Theory of Solidification. Cambridge University Press, UK, 2001.

[101] S. S. L. Peppin, M. G. Worster, and J. S. Wettlaufer. Morphological instability in freezing colloidal suspensions. Proc. Roy. Soc. Lond. A, 463:723-733, 2007.

[102] S. S. L. Peppin, J. S. Wettlaufer, and M. G. Worster. Experimental verification of morphological instability in freezing aqueous colloidal suspensions. Phys. Rev. Lett., 100:238301, 2008 . 
[103] S. S. L. Peppin, A. Majumdar, R. W. Style, and G. J. Sander. Frost heave in colloidal soils. SIAM Journal on Applied Mathematics, 71:1717-1732, 2011.

[104] M. G. Worster. Solidification of fluids, pages 393-446. Perspectives in Fluid Dynamics. Cambridge University Press, Cambridge, UK, 2000.

[105] K. Arakawa. Theoretical studies of ice segregation in soil. Journal of Glaciology, 6:255$260,1966$.

[106] B. Chalmers and K. A. Jackson. Experimental and theoretical studies of the mechanism of frost heaving. CRREL Research Report, 199:1-22, 1970.

[107] T. Ueda and E. Penner. Mechanical analogy of a constant heave rate. In Proceedings of the International Symposium on Frost Action in Soils, pages 57-67, Sweden, 1978.

[108] G. W. Scherer. Freezing gels. Journal of Non-Crystalline solids, 155:11-25, 1993.

[109] E. J. Chamberlain and A. J. Gow. Effect of freezing and thawing on the permeability and structure of soils. Engineering Geology, 13:73-92, 1979.

[110] L. U. Arenson, T. F. Azmatch, and D. C. Sego. A new hypothesis on ice lens formation in frost-susceptible soils. In 9th International Conference on Permafrost, volume 1, pages 59-64, Alaska, 2008.

[111] K. Watanabe, Y. Muto, and M. Mizoguchi. Water and solute distributions near an ice lens in a glass-powder medium saturated with sodium chloride solution under unidirectional freezing. Crystal Growth \& Design, 1:207-211, 2001.

[112] S. A. Shoop and S. R. Bigl. Moisture migration during freeze and thaw of unsaturated soils: modeling and large scale experiments. Cold Regions Science and Technology, 25:3345, 1997.

[113] A. Kade and D. A. Walker. Experimental alteration of vegetation on nonsorted circles: Effects on cryogenic activity and implications for climate change in the arctic. Arctic, Antarctic, and Alpine Research, 40:96-103, 2008. 



\section{RECENT REPORTS}

12/11 Parasite spill-back from domestic hosts may induce an Allee effect in wildlife hosts

Krkošek

Ashander

Lewis

12/12 Modelling temperature-dependent larval development and sub-

Wheeler sequent demographic Allee effects in adult populations of the alpine butterfly Parnassius smintheus

Bampfylde

Lewis

12/13 Putting "space" back into spatial ecology Fortin

Peres-Neto

Lewis

12/14 Wildlife disease elimination and density dependence

Potapova

Merrill

Lewis

12/15 Spreading Speed, Traveling Waves, and Minimal Domain Size in Impulsive Reaction-diffusion Models

Lewis

$\mathrm{Li}$

12/16 MCMC methods for functions modifying old algorithms to make them faster

Cotter

Roberts

Stuart

White

12/17 Weyl Geometry and the Nonlinear Mechanics of Distributed Point Defects

Yavari

Goriely

12/18 A note on oblique water entry

Moore

Howison

Ockendon

Oliver

12/19 Calculus on surfaces with general closest point functions

März

Macdonald

12/20 Multiple equilibria in a simple elastocapillary system

Taroni

Vella

12/21 Multiphase modelling of vascular tumour growth in two spatial dimensions

Hubbard

Byrne

12/22 Chebfun and Numerical Quadrature

Hale

Trefethen

12/23 Moment-based formulation of NavierMaxwell slip boundary con-

Reis ditions for lattice Boltzmann simulations of rarefied flows in microchannels

Dellar

12/24 Correspondence between one- and two-equation models for solute transport in two-region heterogeneous porous media 
12/25 Rolie-Poly fluid flowing through constrictions: Two distinct instabilities

Reis

Wilson

12/26 Age related changes in speed and mechanism of adult skeletal muscle stem cell migration

Collins-Hooper

Woolley

Dyson

Patel

Potter

Baker

Gaffney

Maini

Dash

Patel

12/27 The interplay between tissue growth and scaffold degradation in

ODea engineered tissue constructs

Osborne

El Haj

Byrne

Waters

12/28 Non-linear effects on Turing patterns: time oscillations and chaos.

Aragon

Barrio

Woolley

Baker

Maini

12/29 Colorectal Cancer Through Simulation and Experiment

Kershaw

Byrne

Gavaghan

Osborne

12/30 A theoretical investigation of the effect of proliferation and adhe-

Mirams sion on monoclonal conversion in the colonic crypt

Fletcher

Maini

Byrne

12/31 Convergent evolution of spiny mollusk shells points to elastic en-

Chirat ergy minimum

Moulton

Shipman

Goriely

12/32 Three-dimensional oblique water-entry problems at small dead-

Moore rise angles

Howison

Ockendon

Oliver

12/33 Second weak order explicit stabilized methods for stiff stochastic

Abdulle differential equations

Vilmart

Zygalakis

12/34 The sensitivity of Graphene ‘Snap-through' to substrate geometry

Wagner

Vella 
Copies of these, and any other OCCAM reports can be obtained from:

Oxford Centre for Collaborative Applied Mathematics Mathematical Institute

24 - 29 St Giles'

Oxford

OX1 3LB

England

www.maths.ox.ac.uk/occam 